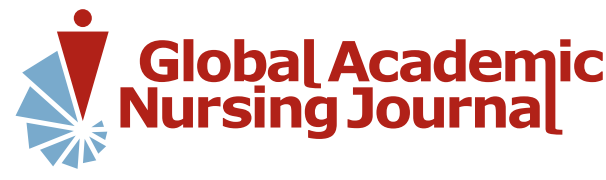

\section{Práticas do enfermeiro no acompanhamento da mãe de recém-nato pré-termo na Atenção Básica}

\author{
Nurses' practices in monitoring the mother of preterm newborns in Primary Care
}

Prácticas de enfermería en el seguimiento de la madre de un recién nacido prematuro en Atención Primaria

\author{
Gisele da Conceição Sabino ${ }^{1}$ \\ ORCID: 0000-0002-3042-298X \\ Denize Mara de Araujo ${ }^{1}$ \\ ORCID: 0000-0002-3783-8136 \\ Maria Regina Bernardo da Silva ${ }^{1}$ \\ ORCID: 0000-0002-3620-3091 \\ Daniel Ribeiro Soares Souza ${ }^{1}$ \\ ORCID: 0000-0002-9170-6193 \\ Nathalia Nunes Gomes ${ }^{1}$ \\ ORCID: 0000-0002-6467-8051 \\ Norival Santolin de Oliveira ${ }^{1}$ \\ ORCID: 0000-0002-1747-0135 \\ Kamille Lopes Formoso \\ Machado ${ }^{1}$ \\ ORCID: 0000-0002-0644-3636
}

${ }^{1}$ Universidade Castelo Branco. Rio de Janeiro, Brasil.

Como citar este artigo:

Sabino GC, Araujo DM, Silva MRB, Souza DRS, Gomes NN, Oliveira NS, Machado KLF. Práticas do enfermeiro no acompanhamento da mãe de recém-nato pré-termo na Atenção Básica. Glob Acad Nurs.

2021;2(Spe.1):e91. https://dx.doi.org/10.5935/26755602.20200091

Autor correspondente: Maria Regina Bernardo da Silva E-mail: m.regina2000@uol.com.br

Editor Chefe: Caroliny dos Santos Guimarães da Fonseca

Editor Executivo: Kátia dos Santos Armada de Oliveira

Submissão: 07-01-2021

Aprovação: 18-01-2021
Resumo

Objetivou-se identificar as práticas dos enfermeiros no acompanhamento das mães de prematuros de baixo peso na Atenção Básica em unidades da Estratégia de Saúde da Família da Zona Oeste AP 5.1 RJ. Realizado um estudo qualitativo de natureza descritiva do tipo pesquisa de campo com 14 enfermeiros atuantes no acompanhamento do recém-nato pré-termo ou baixo peso em três unidades básicas de saúde na Zona Oeste do RJ. Foi abordado com os enfermeiros as possíveis causas relacionadas ao nascimento de bebe prétermo ou com baixo peso, e os fatores intrínsecos e extrínsecos foram, por muitas vezes, identificados durante o acompanhamento de pré natal, onde relatam uso de drogas ilícitas, álcool, tabagismo e outros fatores tais como, hipertensão, diabete que restringem o crescimento uterino e a própria desnutrição materna. É imprescindível que toda equipe participe da educação permanente e esteja voltada para o atendimento à essa mulher neste período tão delicado e frágil. Os profissionais ainda travam um grande desafio de acompanhar essa mãe e esse RN nos primeiros momentos após a alta hospitalar. Observa-se ainda a necessidade de melhoria de fluxo e uma padronização do atendimento.

Descritores: Pré-Termo; Baixo Peso; Mãe; Enfermeiro; Cuidados de Enfermagem.

\section{Abstract}

The aim was to identify the practices of nurses in monitoring the mothers of low weight preterm infants in Primary Care in units of the Family Health Strategy of the West Zone AP 5.1 RJ. A qualitative, descriptive study of the field research type was carried out with 14 nurses working in the monitoring of preterm or low birth weight infants in three basic health units in the West Zone of RJ. The possible causes related to the birth of preterm or low weight babies were discussed with the nurses, and the intrinsic and extrinsic factors were, many times, identified during the prenatal care, where they report the use of illicit drugs, alcohol, smoking and other factors such as hypertension, diabetes that restrict uterine growth and maternal malnutrition itself. It is essential that the entire team participates in permanent education and is focused on assisting this woman in this delicate and fragile period. The professionals still face a great challenge to accompany this mother and this newborn in the first moments after hospital discharge. There is also a need to improve the flow and standardize the service.

Descriptors: Preterm; Low Weight; Mother; Nurse; Nursing Care.

Resumén

El objetivo fue identificar las prácticas de los enfermeros en el seguimiento de las madres de prematuros de bajo peso en Atención Primaria en las unidades de la Estrategia Salud de la Familia de la Zona Oeste AP 5.1 RJ. Se realizó un estudio cualitativo, descriptivo del tipo investigación de campo con 14 enfermeras que laboran en el seguimiento de recién nacidos prematuros o de bajo peso al nacer en tres unidades básicas de salud de la Zona Oeste de RJ. Las posibles causas relacionadas con el nacimiento de bebés prematuros o de bajo peso fueron discutidas con las enfermeras, y los factores intrínsecos y extrínsecos fueron, muchas veces, identificados durante la atención prenatal, donde informan el uso de drogas ilícitas, alcohol, tabaquismo y otros. factores como la hipertensión, la diabetes que restringen el crecimiento uterino y la propia desnutrición materna. Es fundamental que todo el equipo participe de la educación permanente y esté enfocado en ayudar a esta mujer en este delicado y frágil período. Los profesionales aún enfrentan un gran desafío para acompañar a esta madre y a este recién nacido en los primeros momentos tras el alta hospitalaria. También es necesario mejorar el flujo y estandarizar el servicio.

Descriptores: Prematuro; Bajo Peso; Madre; Enfermeiro; Cuidados de Enfermería. 


\section{Introdução}

Conforme dados atuais do Ministério da Saúde, cerca de $10 \%$ dos bebês nascem antes do tempo, sendo considerados prematuros ou pré-termo, ou seja, que vêm ao mundo antes de completar 37 semanas de gestação. Com isso, todo bebe que nasce prematuro, menor que 2500 gramas, é considerado de baixo peso. No Rio de Janeiro, no ano de 2018, dados do sistema de informação DATASUS mostra que tivemos 467 óbitos de recém-nascidos com baixo peso e que a taxa de prematuridade alcançou 5,3\% dos nascidos vivos no Brasil ${ }^{1}$.

Para Carvalho ${ }^{2}$ :

"As prevalências de baixo peso ao nascer encontradas no Brasil, apesar de atingir a meta da cúpula Mundial em favor da Infância, qual seja a de alcançar taxas menores de $10 \%$ dos nascidos vivos, ainda são insatisfatórias, principalmente, se considerarmos que os países desenvolvidos têm taxas em torno de 5\%. Além disso, o baixo peso ao nascer, é um significado indicador da qualidade da assistência à saúde reprodutiva da mulher, haja vista que grande parte de seus fatores causais, como prematuridade, infecções durante a gravidez, tabagismo, gravidez precoce e intervalo interpartal menor que dois anos são na sua maioria, passiveis de controle, com uma atenção adequada a mulher em idade fértil".

Sabe-se que a prematuridade é um dos fatores que contribuem para o crescente número de mortalidade infantil no mundo inteiro e, no Brasil, essa taxa a cada dia vem subindo; causas sociais e econômicas intrínsecas atuam afetando o desenvolvimento da criança. Nesse momento, o profissional de saúde, desde o acompanhamento da gestante no período do parto e na volta dessa mãe para seu domicílio, será de grande importância, pois essa puérpera está em um processo de transição onde será necessário uma reestruturação e reajustamento ao seu novo estilo de vida, sem contar que ela estava preparada para ser mãe e ter logo em seus braços um bebe saudável, o que muitas vezes acontece e essa criança nas primeiras horas de vida não poder estar ao lado da mãe tendo que ficar em incubadora para monitoramento, Nesse período, ela se sente indefesa, desapontada e, às vezes, sem esperanças.

De acordo com a Lei Federal de $n .^{\circ} 7.498 / 86$ e do Decreto-Lei n.o 94.406/87, que decreta o livre exercício da Enfermagem em todo território nacional, é incumbido ao enfermeiro "prestar assistência a gestante, parturiente, puérpera e ao recém-nascido; participar dos programas e atividades de assistência integral a saúde individual e de grupos específicos, particularmente daqueles prioritários e de alto risco" ${ }^{3}$.

A Organização Mundial da Saúde (OMS) tem uma grande preocupação em reduzir a mortalidade infantil desde o período do nascimento, de internação nas neonatais até a ida do recém-nascido para o domicílio. A OMS sabe que uma boa assistência da equipe de saúde minimiza problemas futuros do recém-nascido. A assistência da enfermagem, com a nova política de humanização do SUS, também é fundamental para que essa mãe tenha o entendimento de como o seu bebê é um ser frágil e que merece cuidados
Sabino GC, Araujo DM, Silva MRB, Souza DRS, Gomes NN, Oliveira NS, Machado KLF especiais. Essa mulher, quando bem orientada pelos enfermeiros da Atenção Primária, saberá passar por esse momento difícil em sua vida ${ }^{3,4}$.

Com objetivo de diminuir os índices de mortalidade infantil e o acompanhamento com uma assistência que visasse atender a todos com qualidade, o Ministério da Saúde lançou, em 2004, a Agenda de Compromissos para a Saúde Integral da Criança e Redução de Mortalidade, onde estão as diretrizes de acompanhamentos na atenção à criança nas diversas fases de desenvolvimento, apontando as linhas de cuidados para os recém-nascidos de situação de risco receberem atenção integral na atenção básica, que identificará os aspectos de vulnerabilidade dessa criança e de sua família ${ }^{3,4}$.

Nesse momento em que a puérpera precisa de muito aprendizado e novas informações, é extremamente relevante obter novos conhecimentos e aumentar, ainda mais, o vínculo com os enfermeiros da Clínica da Família ou CMS, onde o binômio mãe-bebê será acompanhado. A equipe precisa entender o grau de conhecimento dessa mãe e dos demais familiares envolvidos, para que assim consigam orientar esse grupo e fazer com que haja uma boa adesão aos cuidados prestados.

Dentro dessa dinâmica, o enfermeiro deve estar qualificado para atender de forma humanizada, conhecer técnicas, como o Método Canguru por exemplo, cuidados no banho e com a alimentação de ambos, procurar entender as reais necessidades do bebê e trabalhar a adaptação da mãe a essa nova realidade no seu cotidiano.

Sabe-se que na maioria dos casos em que o bebê nasceu pré-termo a atenção é mais voltada para esse recémnato. Para tanto, este estudo tem por objetivo geral identificar as praticas dos Eenfermeiros no acompanhamento das mães de prematuros de baixo peso na Atenção Básica em unidades da Estratégia de Saúde da Família da Zona Oeste AP 5.1 RJ.

A justificativa desse estudo se dá pela necessidade de proporcionar conhecimentos e técnicas corretas às mães de bebês prematuros, através dos enfermeiros, a fim de gerar uma melhor adaptação no cuidado realizado no lar do binômio mãe-bebê. É preciso que seja estabelecido um vínculo, oferecendo um cuidado individualizado e com comprometimento para que essa mãe e os demais familiares envolvidos encontrem segurança para dar continuidade ao cuidado do RN após a alta hospitalar e, para que isso ocorra, é preciso que o enfermeiro intervenha nesse processo de interação.

Conforme pesquisador ${ }^{5}$ relata, "[...] a articulação dos cuidados entre os diversos níveis de saúde ainda é tímida, sendo o seguimento destes bebês realizado quase que predominantemente em nível hospitalar". Nessa perspectiva, a pergunta que norteou a pesquisa de estudo foi: Quais são as práticas do enfermeiro no acompanhamento das mães de prematuros de baixo peso na Atenção Básica em unidades da Estratégia de Saúde da Família (ESF) na Zona Oeste do RJ na AP 5.1?

\section{Metodologia}

Foi realizado um estudo qualitativo de natureza 
descritiva do tipo pesquisa de campo sobre as práticas dos enfermeiros no acompanhamento das mães de prematuros de baixo peso na Atenção Básica em unidades da Estratégia de Saúde da Família da Zona Oeste AP 5.1 RJ.

Segundo Minayo ${ }^{6}$, a pesquisa qualitativa:

"[...] busca questões muito específicas e pormenorizadas, preocupando-se com um nível da realidade que não pode ser mensurado e quantificado. Atuam com base em significados, motivos, aspirações, crenças, valores, atitudes, e outras características subjetivas próprias do humano e do social que correspondem às relações, processos ou fenômenos e não podem ser reduzidas à variáveis numéricas".

A pesquisa foi desenvolvida em três unidades da Atenção Básica na Estratégia de Saúde da Família nos bairros de Bangu (Catiri), Realengo e Padre Miguel na AP 5.1 e a escolha das Unidades de Saúde se deu pelo grande volume de atendimento. Foram entrevistados 14 enfermeiros da atenção básica que realizam o acompanhamento mensal de puérperas e de seus respectivos recém-natos prematuros. Foram incluídos os enfermeiros que acompanham o prénatal e estão na unidade há mais de seis meses e excluídos da pesquisa os enfermeiros recém-contratados.

As entrevistas, a princípio, foram realizadas através de formulário no Google Forms - devido ao cenário atual por conta da pandemia da COVID-19 - que foi enviado através de e-mail à unidade de saúde e aos profissionais onde as respostas foram encaminhadas para o e-mail do pesquisador no período de 06 de setembro a 20 de outubro de 2020, logo após a autorização do Comitê de Ética em Pesquisa da Universidade Castelo Branco (CEP/UCB/RJ) sob n.으 4.230.101.

Para a analise do conteúdo das respostas dos participantes, optou-se pela Análise de Bardin 4 , que "[...] é um método muito empírico, dependente do tipo de 'fala' a que se dedica e do tipo de interpretação que se pretende como objetivo".

Para participar desta pesquisa, os sujeitos foram esclarecidos que todos os dados obtidos seriam mantidos em sigilo e privacidade. O Termo de Consentimento Livre e Esclarecido foi assinado por cada participante, atendendo ao que pede a Resolução n. $\mathbf{4}$ 466/12 do Conselho Nacional de Saúde.

\section{Resultados e Discussão}

Participaram da pesquisa 14 profissionais enfermeiros que atuam na Estratégia da Família na Zona Oeste do Rio de Janeiro, na AP 5.1, sendo 03 enfermeiros da Unidade Bangu, 05 da Unidade Padre Miguel e 06 da Unidade Realengo. 42,9\% dos entrevistados possui entre $4 \mathrm{e}$ 6 anos trabalhando na atenção básica e $28,6 \%$ atua de 7 a 10 anos; desses profissionais, 02 atuam em outras áreas, tendo visto que a maioria dos entrevistados possuui uma grande experiência na atenção básica, onde certamente vivenciam as mudanças que vem ocorrendo com o passar dos anos em relação à assistência, podendo justificar a grande procura desses profissionais por especializações.

A grande maioria dos profissionais possui alguma especialização de pós-graduação, $85,7 \%$ optou por se especializar em áreas como gestão em saúde, enfermagem sanitarista, obstetrícia, mas a maioria - em torno de $89,1 \%$ resolveu fazer pós-graduação em Estratégia de Saúde da Família por já estar na área e adquirir mais conhecimento. Sabe-se que nos dias de hoje o mercado de trabalho está muito competitivo e os profissionais da área da saúde tendem a diferenciar se quando possuem alguma especialização em se falando em Estratégia de Saúde da Família, a qual abrange cerca de $63,45 \%$ da população do Brasil, e ainda está em crescimento em algumas regiões que ainda não foram implementadas essa estratégia?

Quadro 1. Perfil dos enfermeiros de três Unidades Básicas da Zona Oeste. Rio de Janeiro, RJ, Brasil, 2020

\begin{tabular}{|c|c|c|c|c|}
\hline Entrevistado & Unidade & $\begin{array}{c}\text { Tempo de Estratégia } \\
\text { da Família (Anos) }\end{array}$ & $\begin{array}{c}\text { Possui } \\
\text { Especialização }\end{array}$ & Área de Abrangência \\
\hline 1 & Bangu & $7-10$ & Sim & Saúde da Família \\
\hline 2 & Bangu & $4-6$ & Sim & Saúde da Família \\
\hline 3 & Bangu & $7-10$ & Sim & Gestão em Saúde \\
\hline 4 & Padre Miguel & $4-6$ & Sim & Saúde da Família \\
\hline 5 & Padre Miguel & $4-6$ & Sim & Obstetrícia \\
\hline 6 & Padre Miguel & $1-3$ & Sim & Sim daúde Família \\
\hline 7 & Realengo & $7-10$ & Sim & Saúde da Família \\
\hline 8 & Realengo & $4-6$ & Sim & Obstetrícia e Saúde da Família \\
\hline 9 & Padre Miguel & $1-3$ & Sim & Gestão Hospitalar \\
\hline 10 & Padre Miguel & $1-3$ & Não & Sim \\
\hline 11 & Padre Miguel & $4-6$ & Sim & Saúde da Família \\
\hline 13 & Realengo & $1-3$ & Saúde da Família \\
\hline 14 & Realengo & $7-10$ & $4-6$ & \\
\hline
\end{tabular}

Dentro da saúde da família, o Enfermeiro possui autonomia para prestar cuidado a vários tipos de pessoas em diferentes fases da vida e com comorbidades já préexistentes, além de trabalhar com a promoção da saúde e prevenção de doenças. Além disso, acompanha a mulher no cuidado da sua saúde desde quando ela descobre a gestação até o período de puerpério, além de monitorar a saúde do seu bebê com o intuito de reduzir as taxas de mortalidade infantil no primeiro ano de vida.

Conforme priorizado pelo Ministérios da Saúde desde 2015, foi criada a Política Integral à Saúde da Criança, para que ocorresse a necessidade do acompanhamento do 
RN e da puérpera o mais precocemente possível a fim de avaliar as condições de saúde do binômio mãe/bebê $\hat{~}^{8}$. Para que esse cuidado com a puérpera seja previsto, o enfermeiro deve ter um controle de todas as gestantes por ele acompanhado e a data provável do parto para o quanto antes essa mulher e o seu bebê sejam acolhidos.

Segundo pesquisadores ${ }^{9}$, "[...] a busca ativa das puérperas é de responsabilidade de toda a ESF, assim, não se justifica o atraso da visita domiciliar, bem como a enfermeira não está isenta de responsabilidade pela demora da captação ou marcação pelo ACS uma vez que é sua atribuição a coordenação destes".

Em relação a instrumentos de trabalho que facilitem o enfermeiro a identificar em tempo hábil e precocemente a puérpera e o RN parto prematuro, os profissionais foram unânimes em dizer que em sua unidade possuem planilha de controle dessas crianças de risco. Esse é um instrumento que tem o intuito de acompanhar o recém-nascido pré-termo ou de baixo peso; e essa planilha é compartilhada entre as maternidades da região relatando quais crianças nasceram e seu respectivo fator de risco. É através desta planilha que profissional tem como captar o prematuro mais recentemente o possível e a mãe ser encaminhada ao primeiro acolhimento na unidade. Relataram também instrumento próprio que facilita em prever a data provável do parto, assim, essa gestante já começa a ser monitorada antes que ganhar o bebê, sendo que caso a mulher tenha o parto antes das 37 semanas, os profissionais não relataram qual a estratégia que possuem para captar essa puérpera.

\section{Instrumento para acompanhamento de recém-nato pré-} termo nas Unidades Básicas de Saúde na Zona Oeste RJ

$100 \%$ dos entrevistados relataram que existe formulário para acompanhamento dos recém-natos prétermo seguem os relatos abaixo:

"Planilha de bebê de risco e formulário da unidade" (E1 Unidade Bangu).

"Planilha de monitoramento bebê de risco e puérperas com vulnerabilidade" (E6 - Unidade Padre Miguel).

"Planilha bebê de risco da CAP compartilhada com o NASF" (E7Unidade Realengo).

Em relação a esse instrumento, sua funcionalidade tem-se por meio de identificar todos os bebês na área adstrita que nasceram com algum fator de risco, como prevê - Ministério da Saúde, crianças de risco são aquelas que manifestam, pelo menos, um destes critérios: residência em área de risco; baixo peso ao nascer $(<2500 \mathrm{~g})$; recém-nascido (RN) com menos de 37 semanas de idade gestacional; asfixia grave (Apgar < 7 no 50 minuto de vida); internamento ou intercorrência na maternidade ou em unidade de assistência ao RN; ser filho de mãe adolescente (<18 anos); ser RN de mãe com baixa instrução ( $<8$ anos de estudo) e haver história de morte de crianças $<5$ anos na família ${ }^{10}$. Sendo assim, o instrumento ajuda os enfermeiros a identificar, além da prematuridade ou baixo peso, outros fatores de risco para o RN.

Questionou-se aos enfermeiros participantes as causas relacionas ao nascimento de um bebê pré-termo ou com baixo peso, os quais esboçaram fatores intrínsecos e extrínsecos que foram, por muitas vezes, identificados durante o acompanhamento de pré-natal. Sendo estas as mais variadas possíveis causas, como: o uso de drogas ilícitas, álcool, tabagismo, hipertensão, restrição do crescimento uterino devido à diabetes, desnutrição materna. Uns dos fatores mais citados pelos entrevistados foi o absentismo nas consultas, porém, não foi unânime, pois os profissionais realizam busca ativa das gestantes que faltam as consultas mesmo com agendando em tempo oportuno ${ }^{10}$.

\section{Possíveis causas relacionadas ao nascimento de um bebê pré-termo ou com baixo peso}

Segundo os enfermeiros entrevistados, inúmeras são as causas para o RN pré-termo. O absenteísmo de consultas foi o mais relatado $16 \%$, em seguida $15 \%$ usuária de drogas e desnutrição, $8 \%$ tabagismo e restrição de crescimento uterino $7,7 \%$.

Relatos dos entrevistados:

"[...] dependentes químicas, diabetes, tabagista" (E2 - Unidade Bangu).

"[...] devido as inúmeras faltas as consultas, muitas vezes, não conseguimos realizar os exames em tempo adequado e só após o parto é que conseguimos descobrir algumas comorbidades que elas não relatam no pré-natal" (E1O - Unidade Padre Miguel).

"A má nutrição materna, hábitos de vida insatisfatórios, uso de álcool, tabagismo contribuem, a meu ver, em um bebê prematuro ou com baixo peso" (E14 - Unidade Realengo).

O uso de substâncias nocivas à saúde no período gravídico puerperal, como drogas lícitas e ilícitas, deve ser investigado e desestimulado, pois crescimento fetal restrito, aborto, parto prematuro, deficiências cognitivas no concepto, entre outros, podem estar associados ao uso e abuso dessas substâncias"11.

Estudo $^{10}$ relata que o estado nutricional materno, assim como o ganho de peso gestacional, vem sendo foco atual de vários estudos, não apenas pela crescente prevalência dos seus distúrbios, mas sobretudo devido ao seu papel determinante sobre os desfechos gestacionais, "[...] dentre estes destacam-se o crescimento fetal e o peso ao nascer, que podem trazer implicações para a saúde do indivíduo ao longo de sua vida, particularmente, em relação às doenças crônicas não transmissíveis".

Considerando a relevância de estudos sobre o assunto, viu-se que:

"A maioria dos artigos publicados aponta que a não realização do pré-natal se deve, principalmente, a fatores sócio econômicos (baixa renda familiar e escolaridade), de acesso às consultas (local de residência distante do serviço e custo para o deslocamento), de qualidade dos cuidados em saúde e de suporte social. Outros fatores potencialmente relacionados são: idade materna (adolescência e idade mais avançada), não convivência 
com companheiro, uso de álcool ou outras drogas na gravidez, multiparidade, não aceitação da gestação, falta de apoio familiar, contexto social adverso, experiências negativas de atendimento e concepções de descrédito sobre o pré-natal"10.

\section{Sentimento das mães em relação aos recém-natos prematuros}

Os entrevistados relataram que, em relação ao recém-nato prematuro ou baixo peso, houve um consenso com relação ao sentimento expressos em responder que as mães apresentam variados sentimentos em relação ao seu filho e que estão passando por um momento critico, necessitando de orientação, apoio emocional e acompanhamento familiar. Seguem as falas dos profissionais:

\section{"Medo e dúvidas sobre o cuidar do RN e também sobre o desenvolvimento da criança com o passar de meses" (E2 - Unidade Bangu). \\ "Medo da morte de seu bebê e de não poder cuidar melhor" (E6 - Unidade Padre Miguel). \\ "Preocupação com o ganho de peso" (E7 - Unidade Realengo).}

O enfermeiro que realiza esse primeiro acolhimento terá que ter uma escuta humanizada, qualificada e tentar acalmar essa mulher e seus familiares, ocorrendo também na alta hospitalar de ambos. Quando o $\mathrm{RN}$, mesmo sendo prematuro, possui um peso adequado e nenhuma complicação existente, mesmo assim essa mulher possui medos em relação à saúde do seu filho, à amamentação, aos cuidados, pois acredita que seu bebê é frágil e necessita de cuidados específicos. Estudo ${ }^{12}$ alerta para o perigo dos pais dos bebês pré-termo desenvolverem a "síndrome da criança vulnerável", devido a uma percepção distorcida dos pais, que passam a se concentrar mais nas deficiências do que nos recursos presentes no repertório do bebê, eles podem superprotegê-lo e superestimar sua vulnerabilidade.

O nascimento do bebê pré-termo configura-se em uma situação de "crise psicológica" na família, a qual passa a enfrentar uma situação imprevisível e ansiogênica geradora de sentimento de impotência e de estresse, especialmente na mãe. A prematuridade do bebê requer a internação em UTI Neonatal, fator este que interfere negativamente no estabelecimento do vínculo mãe-bebê ${ }^{13}$.

Segue o primeiro atendimento das puérperas de recémnato nas unidades básicas.

\section{Primeiro atendimento da puérpera nas unidades básicas}

O primeiro atendimento da puérpera e do recémnato na unidade básica ao terem alta hospitalar, segundo o protocolo de atendimento, deve ser realizado com agendamento prévio da maternidade para unidade de saúde, onde a puérpera procura o profissional de saúde que está no acolhimento para recepcioná-la. O atendimento é feito pelo enfermeiro e pela equipe do NASF, que é composto pelos seguintes profissionais: nutricionista, fonoaudiólogo, psicólogo e fisioterapeuta.
Observa- se que os profissionais entrevistados não apresentam um fluxo único para o primeiro atendimento deste cliente como relatos abaixo:

"Consulta em livre demanda. Acolhimento binômio mãe-bebê, geralmente com a equipe de enfermagem com acolhimento encaminhado para consulta médica, vacinas, teste do pezinho, orientações para mãe e familiares e agendado o retorno [...] em conjunto com a equipe do NASF" (E2 - Unidade Bangu).

"Normalmente pela equipe de enfermagem, com o acolhimento, encaminhamento para consulta médica, vacinas, teste do pezinho, orientações para a mãe e familiar e agendamento do retorno" (E3 - Unidade Bangu).

“Demanda espontânea" (E5 - Unidade Padre Miguel).

"Primeiro acolhimento com até 07 dias de vida pelo médico. Visita da equipe para orientações" (E13 - Unidade Realengo)

Como visto nas respostas acima, não houve uma coesão, cada unidade possui um fluxo diferenciado, porém, no Caderno de Atenção à Saúde da Criança preconiza-se que a linha de cuidado com a criança na atenção básica tenha o objetivo de expressar um fluxo assistencial seguro para a população infantil e suas famílias, de tal modo que garanta o acesso à rede de saúde dessa clientela, de acordo com as necessidades e especificidades, com o fim de unificar em um eixo norteador as ações de prevenção e promoção à saúde da criança recém-nascida.

\section{Protocolo de atendimento dos recém-natos baixo peso}

O protocolo foi elaborado com a finalidade de padronizar um fluxo de atendimento capaz de possibilitar a organização dos processos de trabalho, o acesso universal aos serviços e a oferta de uma atenção integral, de boa qualidade e com resolutividade, a fim de fortalecer o vínculo dessa puérpera com a equipe de saúde.

Seguem as falas dos enfermeiros:

"Quando se identifica que a mãe esteja com medo, inseguranças orienta-se acompanhamento psicológico faz-se o matriciamento com o NASF, e o recém-nato é encaminhado para nutrição, fonoaudiologia e fisioterapia" (Equipe Realengo).

"É muito comum as mães sentirem-se fragilizadas em relação a cuidar do seu RN em domicílio, e muitas vezes não se acham aptas a cuidar de uma criança tão frágil" (Equipe Padre Miguel).

"A adesão de algumas mães nas primeiras consultas é bem sofrivel, pois em um mês são de 3 a 4 visitas na unidade a fim da pesagem semanal para acompanhar ganho ponderal" (Equipe Bangu).

\section{Profissionais envolvidos no acompanhamento do RN e da puérpera}

O médico vem como o profissional que mais acolhe esse tipo de público $(78,6 \%)$ - bebê e a mãe no grupo de risco com vulnerabilidade -, seguido da nutricionista $14,3 \%$ - esse bebê necessita de ganho de peso, logo, esse profissional é de grande relevância para que se alcance os resultados esperados -, o psicólogo foi citado como 7,1 \% dos entrevistados - muitas vezes essa mãe se sente depressiva, temerosa, frustrada até por não conseguir amamentar seu 
filho e o profissional auxilia, realiza uma escuta humanizada com essa mulher a fim de prevenir danos à saúde mental para poder cuidar do seu bebê.

Em relação ao acompanhamento dessa criança e dessa mãe, as respostas dos profissionais foram sobre recém-natos prematuros quando têm alta do hospital são encaminhados para o acolhimento da unidade básica, caso a mãe não possa ir à unidade, a visita deverá ser domiciliar, que pode também ser realizada pelo médico da Equipe de Saúde da Família; as consultas serão logo que tiver alta depois com 7, 15 e 30 dias para acompanhamento do ganho ponderal de peso desse RN. Dado acompanhamento é preconizado, pois esse bebê e essa mãe necessitam de monitoramento constante no primeiro mês de vida.

Os profissionais relatam muitas faltas sobre a busca ativa dessa mãe, pelo agente comunitário de saúde, algumas vão à maternidade visitar seus bebês que ainda estão na UTI neonatal, outras quando o bebê tem alta e fica em casa de familiares fora da área adstrita com medo de sozinhas não conseguirem cuidar do seu bebê e de si mesma.

"Levam às consultas, mas têm muitas faltas seguidas ou deixam de vir no horário agendado com grandes atrasos" (E1 - Unidade Bangu).

"Geralmente as mães têm uma boa adesão ao acompanhamento, mas a maior dificuldade é no primeiro mês devido ao quantitativo de consultas" (E4 - Unidade Padre Miguel).

"Às vezes sim, devido as mães passarem uma parte do período do puerpério na casa de outro familiar, fora do território. Normalmente levam às consultas sim, embora quando faltam, o ACS faz a busca ativa junto com a equipe, para agendá-los o mais breve possivel" (E2 - Unidade Bangu).

Visto que os profissionais relatam que as mães comparecem, porém, o número de absenteísmo às consultas de acompanhamento ainda é grande, vale destacar que, dentre as ações essenciais da APS, a visita domiciliar é uma estratégia potente por possibilitar uma compreensão ampliada do processo saúde-doença e proporcionar intervenções relacionadas ao cuidado e à manutenção da saúde, tanto do indivíduo quanto da família, perpassando o limite das práticas curativas, e pode ser realizada por qualquer profissional, não somente o agente de saúde ${ }^{14}$.

Sendo assim um ponto relevante para que os profissionais enfermeiros também pudessem realizar a busca ativa quando possível, conscientizando a mãe a não faltar as consultas de acompanhamento do seu bebê, pois o mesmo inspira cuidados especiais nos primeiros meses de vida.

\section{Educação em saúde entre os enfermeiros}

Em relação ao programa educativo para orientação dessas mulheres com recém-natos prematuros, 64,3\% dos enfermeiros informaram que realiza ações educativas, e $35,7 \%$ não estava fazendo no momento por conta do cenário atual da pandemia.

Foi enfatizado pelos entrevistados a necessidade da melhoria na educação em saúde para a puérpera de RN baixo peso sendo, seguida de realizar consultas de pré-natal com orientações da equipe multidisciplinar e individual referente a cuidados sobre amamentação e de higiene. Os entrevistados relataram a necessidade de acompanhamento multiprofissional com profissionais treinados para monitoramento dessas mulheres tão fragilizadas e com necessidade de informação.

Isso reforça o que foi pactuado na primeira Conferência Internacional sobre Promoção de Saúde e que está documentada na Carta de Ottawa, que diz que:

"A promoção da saúde tem, portanto, estreita relação com a educação em saúde que se estabelece a partir da participação da população, de suas necessidades, de seu estilo de vida, crenças e valores, desejos, opções, vivências, da subjetividade e intersubjetividade, no contexto cultural - sócio - político em que vive. Essa participação exige envolvimento, compromisso e solidariedade, enquanto uma construção cotidiana de decisões em conjunto, estabelecidas com todos que participam do processo educativo, que mantêm o compromisso de trocar experiências, vivências, conhecimentos, que são diferentes porque as histórias de cada um foram construídas diferentemente"15.

Em relação à educação em saúde, seguem relatos:

"É oferecido toda orientação geral quanto aos cuidados com o $R N$, no intuito de melhor bem-estar, tanto no domicílio, quanto no acompanhamento da saúde em outras redes de apoio, não só em nossa unidade, mas quando necessário encaminhamos a outros especialistas para prosseguimento do cuidado integral" (E3 - Unidade Bangu).

"Acredito que a enfermagem poderia ter mais curso de atualização e humanização com esse tipo de paciente e fazer mais atendimentos individuais" (E6 - Unidade Padre Miguel).

Os discursos dos profissionais permitiram identificar fragilidades em relação a ações voltadas para educação em saúde. Grande parte já realiza nas consultas de pré-natal, em grupos de gestantes, porém poucos relataram o cuidado voltado para a mulher após o parto, no caso mais aprimorado para as mulheres mães de RN prematuro e baixo peso.

Surge então a necessidade de uma ação conjunta das unidades com os gestores para uma educação permanente voltada para essas mães e seus bebês, onde esses profissionais seriam capacitados para atuar com expertise atentando para a diminuição da morbimortalidade infantil no primeiro ano de vida. Observa-se dificuldades no processo educativo, onde o profissional deve não só promover a saúde visando o coletivo, mas o individual, ainda se tratando de uma puérpera com seu bebê pré-termo, cuja qual deverá ser muito bem orientada desde o momento que adentra a unidade de saúde, quanto à questão do vínculo como o fator principal para que haja a melhoria do acompanhamento e desenvolvimento do convívio entre a mãe e o RN.

\section{Conclusão}

Em virtude dos fatos mencionados na pesquisa com 
Práticas do enfermeiro no acompanhamento da mãe de recém-nato pré-termo na Atenção Básica Sabino GC, Araujo DM, Silva MRB, Souza DRS, Gomes NN, Oliveira NS, Machado KLF

os profissionais enfermeiros que atuam no acompanhamento das puérperas de RN pré-termo ou baixo peso, podemos levar em consideração a importância desse profissional e de sua qualificação na assistência, no acompanhamento e nos resultados positivos que ele e sua equipe possam ter com essa mãe e esse bebê. Em vista dos argumentos apresentados, foi relevante ver que o enfermeiro precisa do apoio de profissionais de outras áreas para que essa mulher e esse bebê tenham um atendimento de excelência ou qualidade. É imprescindível que toda equipe participe da educação permanente e esteja voltada para o atendimento a essa mulher neste período tão delicado e frágil. Conclui-se que os profissionais ainda travam um grande desafio de acompanhar essa mãe e esse RN nos primeiros momentos após a alta hospitalar. Observase ainda a necessidade de melhoria de fluxo e uma padronização do atendimento.

\section{Referências}

1. Ministério da Saúde (BR). Política nacional de atenção integral a saúde da criança - orientações para implementação. Brasília (DF): MS; 2018.

2. Carvalho MR, Prochnik M. Método mãe canguru de atenção ao prematuro. Rio de Janeiro: BNDES; 2001.

3. Conselho Federal de Enfermagem. Decreto-Lei n. 94.406/87. Regulamenta a Lei no 7.498, de 25 de junho de 1986, que dispõe sobre o exercício da Enfermagem, e dá outras providências. Brasília (DF): COFEN. 1987.

4. Bardin L. Análise de conteúdo. São Paulo. Edições 70; 2011.

5. Custódio N, Abreu FCPD, Marski BDSL, Mello DFD, Wernet M. Alta da unidade de cuidado intensivo neonatal e o cuidado em domicílio: revisão integrativa da literatura. Reme Rev Min Enferm. 2013 out/dez;17(4). DOI: 10.5935/1415-2762.20130071

6. Minayo M. (Org) Pesquisa Social, Teoria Método e Criatividade. 29. Ed. Petrópolis: Vozes; 2010.

7. Cascaes AM, Gauche H, Baramarchi FM, Borges CM, Peres KG. Prematuridade e fatores associados no Estado de Santa Catarina, Brasil, no ano de 2005: análise dos dados do Sistema de Informações sobre Nascidos Vivos. Cad Saude Pública. 2008; 24(5):1024-32. DOI: 10.1590/S0102-311X2008000500009

8. Ministério da Saúde, Secretária de Atenção à Saúde (BR). Atenção humanizada ao recém-nascido de baixo peso: Método canguru. 2. ed. Brasília (DF): MS; 2013.

9. Silva CR, Andrade DNP, Ostermann AC. Análise da Conversa: Uma breve Introdução. Revel [Internet]. 2009 [acesso em 20 abr 2020];7(13):1-21. Disponível em: https://webcache.googleusercontent.com/search?qq

10. Zadik Z. Maternal nutrition, fetal weight, body composition and disease in later life. J Endocrinol Invest. 2003; 26:941-61. DOI: 10.1007/BF03345248

11. Passini Júnior R. Consumo de álcool durante a gestação. Rev Bras Ginecol Obstet. 2005;27(7):373-5. DOI: 10.1590/S010072032005000700001

12. Brazelton TB. Momentos decisivos do desenvolvimento infantil. (J.L.Camargo, Trad.) São Paulo: Martins Fontes; 1994.

13. Kennel JH, Klaus MH. Atendimento para os pais de bebês prematuros ou doentes. Em: M.H. Klaus \& J.K. Kennel. Pais/Bebês - a formação do apego (D. Batista, Trad.). Porto Alegre: Artes Médicas; 1993.

14. Nascimento JS, Costa LMC, Santos RM, Anjos DS. Visitas domiciliares como estratégias de promoção da saúde pela enfermagem. Rev Bras Promoção Saúde. 2013; 26(4):513-522. DOI: 10.5020/18061230.2013.p513

15. Promoção da Saúde Carta de Ottawa, Declaração de Adelaide, Declaração de Sundsvall, Declaração de Bogotá. Brasília, DF: [s.n.], 1996. 